\title{
Solid State Pathways towards Molecular Complexity in Space
}

\author{
Harold Linnartz ${ }^{1}$ Jean-Baptiste Bossa $^{1}$, Jordy Bouwman ${ }^{1 \dagger}$, \\ Herma M. Cuppen ${ }^{1 \ddagger}$, Steven H. Cuylle ${ }^{1}$, Ewine F. van Dishoeck ${ }^{1,2}$, \\ Edith C. Fayolle ${ }^{1}$, Gleb Fedoseev ${ }^{1}$, Guido W. Fuchs ${ }^{1}$, Sergio Ioppolo ${ }^{1}$, \\ Karoliina Isokoski $^{1}$, Thanja Lamberts ${ }^{1}$, Karin I. Öberg ${ }^{1}$, \\ Claire Romanzin ${ }^{1} \|$, Emily Tenenbaum ${ }^{1}$, Junfeng Zhen ${ }^{1}$ \\ ${ }^{1}$ Sackler Laboratory for Astrophysics, Leiden Observatory, University of Leiden, PO Box 9513, \\ NL 2300 RA Leiden, the Netherlands \\ email: linnartz@strw.leidenuniv.nl \\ ${ }^{2}$ Max-Planck-Institut für Extraterrestrische Physik, Giessenbachstrasse 1, D-85741 Garching, \\ Germany
}

\begin{abstract}
It has been a long standing problem in astrochemistry to explain how molecules can form in a highly dilute environment such as the interstellar medium. In the last decennium more and more evidence has been found that the observed mix of small and complex, stable and highly transient species in space is the cumulative result of gas phase and solid state reactions as well as gas-grain interactions. Solid state reactions on icy dust grains are specifically found to play an important role in the formation of the more complex "organic" compounds. In order to investigate the underlying physical and chemical processes detailed laboratory based experiments are needed that simulate surface reactions triggered by processes as different as thermal heating, photon (UV) irradiation and particle (atom, cosmic ray, electron) bombardment of interstellar ice analogues. Here, some of the latest research performed in the Sackler Laboratory for Astrophysics in Leiden, the Netherlands is reviewed. The focus is on hydrogenation, i.e., $\mathrm{H}$-atom addition reactions and vacuum ultraviolet irradiation of interstellar ice analogues at astronomically relevant temperatures. It is shown that solid state processes are crucial in the chemical evolution of the interstellar medium, providing pathways towards molecular complexity in space.
\end{abstract}

Keywords. astrochemistry, infrared: Astrochemistry, ISM, ISM: atoms, ISM: molecules, molecular processes, ISM: abundances, infrared: ISM, methods: laboratory, techniques: spectroscopic

\section{Introduction}

At the low temperatures in space, micrometer sized dust grains act as small cryopumps onto which gas-phase molecules accrete. In dense cores, the approximate timescale at which gas-phase species deplete-out onto grains is about $10^{5}$ years - shorter than the typical lifetime of a dense core - and hence, during the first stage of star formation virtually all species (except $\mathrm{H}_{2}$ ) are expected to be frozen-out onto interstellar grains. The resulting ice coating provides a molecule reservoir, comprising tens of monolayers (ML)

$\dagger$ Present address: Lawrence Berkeley National Laboratory, 1 Cyclotron Road, MS 6R-2203 Berkeley, CA 94720-8226 USA

$\ddagger$ Present address: Institute for Molecules and Materials (IMM), Radboud University Nijmegen, PO Box 9010, NL 6500 GL Nijmegen, The Netherlands

I Present address: Harvard-Smithsonian Center for Astrophysics, 60 Garden Street, MS 42, Cambridge, MA 02138 USA

\| Present address: LPMAA, Université Pierre et Marie Curie, 4 place Jussieu, F 75252 Paris cedex, France 
of accreted molecules, and a heat sink capable of absorbing reaction excess energy, effectively catalyzing surface reactions. Basically, inter- and circumstellar surfaces offer an environment where atoms and molecules accrete, meet and greet. Grain surface chemistry is governed by the accretion rate of gas-phase species onto the grains, the surface migration rate, which sets the reaction network, and the desorption rate. I.e., it depends on a number of physical parameters, such as ice temperature and ice morphology. External triggers are needed to overcome reaction barriers and to initiate chemical processes in the ice (i.e., after accretion and before desorption), e.g., by photon irradiation - particularly Ly- $\alpha$ and x-rays - thermal effects and particle bombardment $(\mathrm{H}, \mathrm{D}, \mathrm{C}, \mathrm{N}, \mathrm{O}, \mathrm{S}$ atoms, free electrons as well as cosmic rays). In order to interpret the relevance of these processes in space, it is necessary to fully understand the physical and chemical processes that are at play and for this detailed laboratory experiments are a prerequisite. This is the topic of the present proceeding that is organized in the following way. It starts with the spectral features of interstellar ices and their laboratory analogues. An example is given for $\mathrm{CO}$ ice mixed with different constituents, and summarizing the findings from a very recent paper (Cuppen et al. 2011). It continues with surface processes induced by $\mathrm{H}$-atom addition reactions and vacuum ultraviolet irradiation, and concludes with experiments studying molecular complexity in ice. The hydrogenation and UV irradiation part of this proceeding are based on highlights presented by Ioppolo et al. 2011c, and in a upcoming book chapter on solid state laboratory astrophysics.

\section{Ice spectroscopy}

Spectral features of ices in star-forming regions have been observed particularly in midinfrared absorption studies of embedded objects or along the line of sight of a background star. Ground-based observations in the mid-IR spectral range are limited largely to the 35 micron range because of telluric absorptions, so airborne and space observations have been used to identify solid-state species at longer wavelengths. Observations with the Infrared Space Observatory (ISO) have improved our understanding of interstellar ices substantially. Because of its limited sensitivity, ISO observed mostly bright sources, such as high-mass young stellar objects (YSOs) and quiescent dense clouds toward luminous background stars (Gibb et al. 2000, 2004). More recently, the Spitzer Space Telescope characterized the molecular content of icy grain mantles in the $5-35 \mu \mathrm{m}$ wavelength range towards more than 40 low-mass protostars within the $\mathrm{c} 2 \mathrm{~d}$ (cores to disks) program (e.g., Boogert et al. 2008; Pontoppidan et al. 2008; Öberg et al. 2008; Bottinelli et al. 2010) and dozens more within other programs (Zasowski et al. 2009). The Spitzer data are complemented by ground-based 3-5 micron surveys of the same sources (e.g., Pontoppidan et al. 2003, van Broekhuizen et al. 2005). Following the outcome of dedicated laboratory studies (Hudgins et al. 1993), this has made possible the unambiguous identification of a series of solid state constituents, like $\mathrm{H}_{2} \mathrm{O}, \mathrm{CO}_{2}, \mathrm{CO}, \mathrm{CH}_{4}, \mathrm{CH}_{3} \mathrm{OH}$, and $\mathrm{NH}_{3}$, as well as their likely mixing ratios (see e.g., Bouwman et al. 2009). As ice matrix interactions are different for different ice morphologies, peak positions, band widths and intensity ratios differ for these features and this allows quite accurate comparisons between astronomical and laboratory spectra (Tielens 1991). The latter are typically recorded in Fourier Transform InfraRed (FTIR) transmission experiments of ices deposited at different mixing ratios and studied in full dependence of the temperature. An interstellar ice feature inventory with abundances relative to water ice towards dark clouds, low- and high-mass young stellar objects is given by Öberg et al. (2011).

These are the basic ingredients taken for the laboratory recipes (described in the next sections) to form new molecules. Besides information on the actual chemical composition 


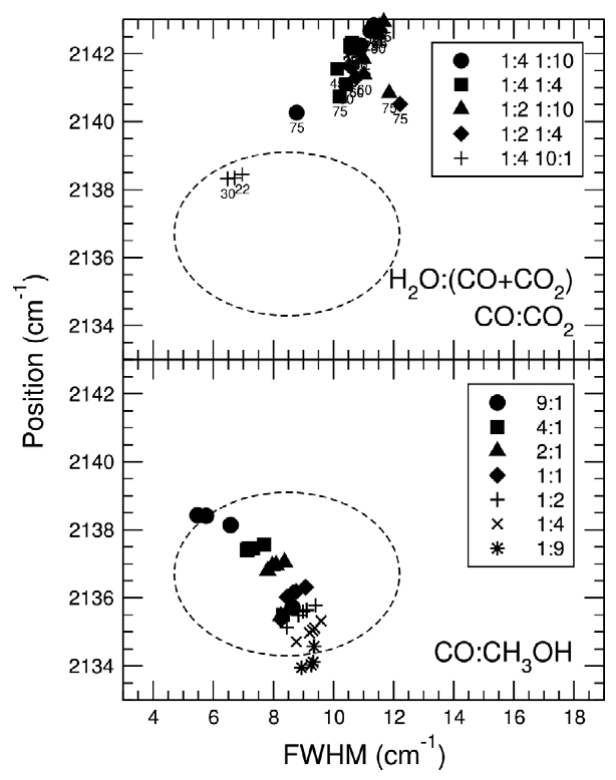

Figure 1. The FWHM and position of the $\mathrm{CO}$ absorption band in laboratory spectra of a range of different $\mathrm{H}_{2} \mathrm{O}: \mathrm{CO}: \mathrm{CO}_{2}$ mixtures (top panel) and $\mathrm{CO}: \mathrm{CH}_{3} \mathrm{OH}$ (bottom panel) ice mixtures at different temperatures. The circles indicate the observational data position within the panels. Figure from Cuppen et al. (2011).

of ices in space, accurate comparisons between astronomical and laboratory data also provides information on the chemical history of the ice. This is illustrated for a specific case in Section 2.1.

\section{1. $\mathrm{CO}$ ice mixed with $\mathrm{CH}_{3} \mathrm{OH}$ ice: a proof for methanol ice residing in a water poor environment}

In observations towards icy sources, the CO stretching band comprises a prominent and broad component around $2136.5 \mathrm{~cm}^{-1}$. This feature is generally attributed to solid CO mixed in a hydrogen-bonded environment like water, but $\mathrm{CO}: \mathrm{H}_{2} \mathrm{O}$ laboratory studies have not been able to fully reproduce this feature. Water-containing $\mathrm{CO}$ ice cannot reproduce the observed band position and bandwidth without simultaneously producing a shoulder at $2152 \mathrm{~cm}^{-1}$. This band, believed to originate from the interaction of dangling$\mathrm{OH}$ bonds with $\mathrm{CO}$, is not observed in any of the astronomical spectra. It has been suggested that the $2152 \mathrm{~cm}^{-1}$ feature is suppressed in astronomical ices by blocking of the dangling $\mathrm{OH}-$ bonds by other species (Fraser et al. 2004). A recent spectroscopic study of $\mathrm{H}_{2} \mathrm{O}: \mathrm{CO}: \mathrm{CO}_{2}$ mixtures (Cuppen et al. 2011) - given the abundances listed by Öberg et al. (2011) a logical choice - shows that such an ice mixture does not offer an explanation for this discrepancy between astronomical and laboratory data. This is illustrated in the upper part of the Fig. 1. Even though the $2152 \mathrm{~cm}^{-1}$ band can be suppressed under low temperature conditions, it is not possible to reproduce peak position and band width of the $2136.5 \mathrm{~cm}^{-1}$ band at the same time. This is indicated by the circle that defines boundary conditions as derived from interpreting the astronomical data, and clearly the spectroscopic parameters for most of the studied mixing ratios are outside this circle. An ice mixture containing only $\mathrm{CO}$ and $\mathrm{CH}_{3} \mathrm{OH}$ and no $\mathrm{H}_{2} \mathrm{O}$, however, does reproduce the spectra at low temperature, both in terms of peak position and band width of the $\mathrm{CO}$ component and the $2152 \mathrm{~cm}^{-1}$ band does not appear. This is illustrated for seven different mixing ratios in the lower panel of the figure. This provides additional proof that $\mathrm{CO}$ only freezes out after $\mathrm{H}_{2} \mathrm{O}$ has formed on the grain, leading to at least two 
separate ice components; a $\mathrm{H}_{2} \mathrm{O}$ rich and a $\mathrm{CO}$ rich one. In this scenario, the spectral characteristics of the $\mathrm{CO}$ central feature require a high fraction of $\mathrm{CH}_{3} \mathrm{OH}$ to be present. This means that almost all methanol is present in the $\mathrm{CO}: \mathrm{CH}_{3} \mathrm{OH}$ component. This is in general agreement with astronomical observations of solid $\mathrm{CH}_{3} \mathrm{OH}$, also indicating that at least part of the methanol is in a water poor environment.

The observation that $\mathrm{CH}_{3} \mathrm{OH}$ is predominantly mixed with $\mathrm{CO}$ in a $\mathrm{H}_{2} \mathrm{O}$-poor environment leads to the conclusion that methanol is mainly formed through hydrogenation of CO, as shown by Watanabe et al. (2004) and Fuchs et al. (2009) and not through $\mathrm{UV}$ or ion processing of a $\mathrm{H}_{2} \mathrm{O}$ containing ice. Grain surface chemistry simulations of $\mathrm{CO}$ in a water-poor environment indeed show that methanol can be formed in this way and that $\mathrm{CH}_{3} \mathrm{OH}$ is mainly present on the grain mantles in pure form or mixed with $\mathrm{CO}$, and that it is not in a water-rich phase (Cuppen et al. 2009). Further details of this work are available from Cuppen et al. (2011). The main conclusion is that not all species are intimately mixed in the ice and that the composition of astrophysically relevant ice mixtures should therefore be treated with care. Within this context it is also relevant that ice segregation can take place. For separate studies see Öberg et al. (2009e) and Fayolle et al. (2011a).

\section{Hydrogenation reactions in interstellar ice analogues}

The first realistic gas-grain model was proposed by Tielens \& Hagen (1982). Their astrochemical model includes a complex grain surface reaction network to explain molecule formation in quiescent dark clouds. According to this model in a first phase, $\mathrm{H}_{2} \mathrm{O}$ ice can be produced by the sequential hydrogenation of $\mathrm{O}$ atoms landing on the grain. Atomic oxygen can also react with other $\mathrm{O}$ atoms to form $\mathrm{O}_{2}$ and $\mathrm{O}_{3}$. Reaction of $\mathrm{O}_{3}$ with $\mathrm{H}$ reforms $\mathrm{O}_{2}$ and $\mathrm{OH}$. $\mathrm{O}_{2}$ can be hydrogenated to form $\mathrm{H}_{2} \mathrm{O}$, while the $\mathrm{OH}$ radical can react with $\mathrm{H}$ or $\mathrm{H}_{2}$ to form again $\mathrm{H}_{2} \mathrm{O}$, or it can form $\mathrm{CO}_{2}$ and $\mathrm{H}_{2}$ with the $\mathrm{CO}$ accreted on the surface. Since hydrogen is the most abundant component of the interstellar gas, this first phase of grain-surface chemistry results in the formation of a polar ice (waterrich) mantle onto the dust grains. During the second phase, when the density increases in the molecular cloud, CO freezes-out onto the grains (its accretion rate is higher than that of $\mathrm{H}$ ) forming an apolar (water-poor) ice layer on top of the polar one. Under these conditions, the hydrogenation of $\mathrm{CO}$ ice leads to the formation of $\mathrm{H}_{2} \mathrm{CO}$ and $\mathrm{CH}_{3} \mathrm{OH}$ (Tielens \& Hagen 1982; Charnley et al. 1997). $\mathrm{CO}_{2}$ can be formed through the reaction $\mathrm{CO}+\mathrm{OH}$ (Goumans et al. 2008). As suggested by Charnley et al. (2001), the ongoing reaction between $\mathrm{CO}$ or $\mathrm{CO}$ hydrogenation reaction products and elements heavier than $\mathrm{H}$ may lead to the formation of more complex molecules such as $\mathrm{CH}_{3} \mathrm{COH}$ and $\mathrm{C}_{2} \mathrm{H}_{5} \mathrm{OH}$ (see reaction diagrams in Charnley \& Rodgers 2005). These and other astrochemical reaction networks were based on chemical intuition and analogues from gas-phase routes. It took several decades before experimental techniques allowed laboratory astrochemists to put all these reactions to the test in the solid state.

Typical experiments are performed using Ultra High Vacuum (UHV) setups with pressures well below $10^{-10}$ mbar, such as our SURFace REaction Simulation DEvice (SURFRESIDE), which comprises a cryogenic unit where ices are grown for astronomically relevant temperatures and a H-atom beam line. A schematic view of the experimental apparatus is shown in Fig. 2.

Ices are monitored by means of Reflection-Absorption InfraRed Spectroscopy (RAIRS) using a Fourier Transform InfraRed (FTIR) spectrometer, which covers the range between 4000 and $700 \mathrm{~cm}^{-1}(2.5-14 \mu \mathrm{m})$. A spectral resolution between 1 and $4 \mathrm{~cm}^{-1}$ is used and several scans are co-added. Two different procedures are used; the ice is first deposited and then hydrogenated/deuterated (see e.g., Fuchs et al. 2009; Ioppolo et al. 2011b, and 


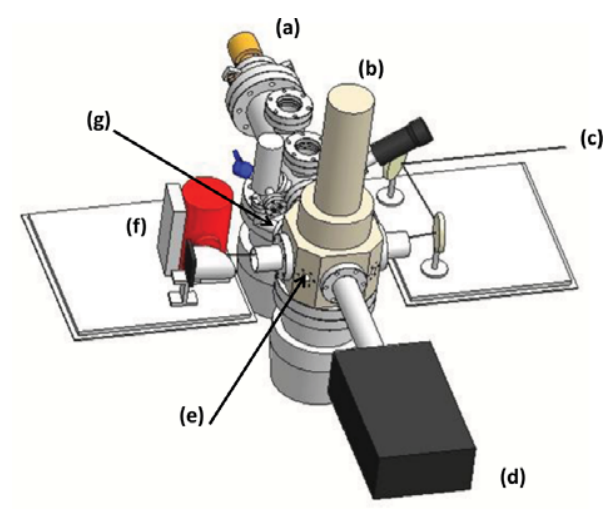

Figure 2. Schematic top-view of the solid-state experimental UHV setup (SURFRESIDE): ${ }^{(a)}$ H-atom source; ${ }^{(b)}$ cold finger; ${ }^{(c)}$ IR beam; ${ }^{(d)}$ mass spectrometer; ${ }^{(e)}$ main chamber; ${ }^{(f)} \mathrm{IR}$ detector; ${ }^{(g)}$ deposition line.

Romanzin et al. 2011) - in this case, RAIR difference spectra with respect to the initial deposited ice are acquired during H/D exposure - or molecules are co-deposited with $\mathrm{H}$ atoms and RAIR difference spectra are acquired with respect to the bare substrate during co-deposition (Cuppen et al. 2010). In all cases, newly formed solid species are monitored by using unique IR spectral signatures. Spectra are recorded at different stages during hydrogenation, providing time resolved information about the destruction (i.e., use-up) of the precursor ice (the deposited ice layer) and the formation of new molecules that are identified through their spectral fingerprints. The intensity of a spectrum can be translated into a column density using a modified Lambert-Beer equation (Bennett et al. 2004). At the end of the H-atom addition a Temperature Programmed Desorption (TPD) experiment can be performed to constrain the spectroscopic results. Surface hydrogenation reactions of simple ices, like pure $\mathrm{CO}, \mathrm{O}_{2}, \mathrm{O}_{3}$, and $\mathrm{CO}: \mathrm{O}_{2}$ mixtures are investigated for a full range of different laboratory conditions including $\mathrm{H} / \mathrm{D}$-atom fluxes, ice temperatures, ice thicknesses, ice structures, and mixture ratios. This makes it possible to unreveal the physics and chemistry of molecule formation and where applicable to examine in more detail the astronomical implications.

In the past the chemistry of inter- and circumstellar ice analogues has been studied using a top-down scenario: icy mixtures of astronomical constituents with more or less realistic mixing ratios were chemically triggered through $\mathrm{UV} /$ cosmic ray irradiation. The resulting residue was shown by several groups to contain complex organic compounds. The experimental results using this approach have been compared to interstellar ices generally in a more qualitative than quantitative way. More recently, a bottom-up approach has become experimentally possible, through the use of the aforementioned UHV setups in which individual reactions of simpler ices (i.e., not the cumulative outcome of a chemical network in ice mixtures) can be studied in situ and in real time under fully controlled laboratory conditions. This approach makes it possible to derive fundamental and molecule specific parameters, like reaction rates and diffusion barriers, which can then be included in astrochemical models to simulate the ice evolution under much longer timescales $\left(10^{5} \mathrm{yr}\right)$ than accessible in the laboratory $(<1$ day).

Here we follow a bottom-up approach and summarize a representative sample of relevant experiments mainly by our group (see e.g., Fuchs et al. 2009; Ioppolo et al. 2008, 2010a, 2011a,b; Cuppen et al. 2010; Romanzin et al. 2011), but it should be explicitly stated that other groups - such as the groups of Watanabe, Lemaire and Dulie - are very active in this field as well. These experiments prove that species like $\mathrm{H}_{2} \mathrm{CO}, \mathrm{CH}_{3} \mathrm{OH}$ 


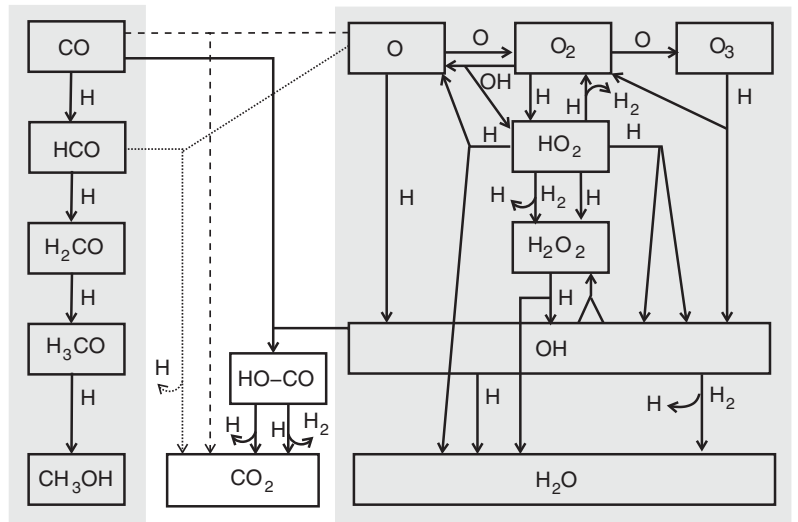

Figure 3. Schematic representation of the hydrogenation reactions in $\mathrm{CO}, \mathrm{O} / \mathrm{O}_{2} / \mathrm{O}_{3}$ and $\mathrm{CO}: \mathrm{O}_{2}$ mixed ice.

and $\mathrm{H}_{2} \mathrm{O}$ can be formed at low temperatures by simple hydrogenation (i.e., without the need for thermal, UV or cosmic ray processing) and provide the basic molecular data to simulate their formation on astronomical timescales (e.g., Cuppen et al. 2009), even though the ice as a whole is not fully representative for a realistic astronomical ice.

Figure 3 shows the schematic representation of the reaction network that is discussed here. The formation of formaldehyde and methanol ice through the $\mathrm{CO}+\mathrm{H}$ channel is shown on the left-side of the figure, while the water formation channels $\left(\mathrm{O} / \mathrm{O}_{2} / \mathrm{O}_{3}+\mathrm{H}\right)$ are plotted on the right-side. The possible $\mathrm{CO}_{2}$ formation routes in a $\mathrm{CO}: \mathrm{O}_{2}$ mixed ice are shown in between the aforementioned channels.

\subsection{Surface formation of methanol}

The surface formation of formaldehyde $\left(\mathrm{H}_{2} \mathrm{CO}\right)$ and methanol $\left(\mathrm{CH}_{3} \mathrm{OH}\right)$ by hydrogenation of $\mathrm{CO}$ ice has been topic of several studies (e.g., Hiraoka et al. 2002; Watanabe \& Kouchi 2002; Fuchs et al. 2009). Methanol has been observed in interstellar ices and has been experimentally shown as a good starting point for the formation of more complex species (Öberg et al. 2009c). The hypothesis that interstellar methanol forms in the solid phase has been recently supported by experimental and modelling work, showing that the gas-phase route via ion neutral reactions is less efficient than previously assumed (Geppert et al. 2005; Garrod et al. 2006). However, the first studies on solid CO hydrogenation by two different groups yielded conflicting results: in one study (Hiraoka et al. 2002) only the formation of $\mathrm{H}_{2} \mathrm{CO}$ was reported, whereas in the other study also $\mathrm{CH}_{3} \mathrm{OH}$ was observed (Watanabe \& Kouchi 2002). The prevailing discrepancy between these studies was experimentally shown by Fuchs et al. (2009) to be a consequence of different experimental conditions, most noticeable the adopted H-atom fluxes and they were also able to determine reaction rates from RAIR data for different ice temperatures and ice thicknesses, as well as H-atom fluxes.

The formation of $\mathrm{H}_{2} \mathrm{CO}$ and $\mathrm{CH}_{3} \mathrm{OH}$ in the ice was also confirmed mass spectrometrically. The experiments showed that the hydrogenation process is thickness independent for layers thicker than 4 monolayers, which represents the maximum penetration depth of the $\mathrm{H}$ atoms into the $\mathrm{CO}$ ice. For temperatures higher than $15 \mathrm{~K}$, a clear drop in the production rate of methanol is observed. This is probably due to the reduced residencetime of $\mathrm{H}$ atoms in the ice at those temperatures, which decreases the probability that hydrogenation reactions occur. Since the rate of formation of molecules depends on a sequence of events: deposition of $\mathrm{H}$ atoms, diffusion and then reaction, reaction barriers can only be determined using a detailed model to fit the experimental data. These results 
can then be used in an astrochemical model to investigate the formation of formaldehyde and methanol under interstellar conditions (Cuppen et al. 2009).

\subsection{Surface formation of water}

Tielens \& Hagen (1982) proposed that interstellar water forms on grain surfaces through three reaction channels: hydrogenation of atomic and molecular oxygen and ozone. Using a Monte Carlo approach, Cuppen \& Herbst (2007) and Cazaux et al. (2010) showed that the contribution of the different formation channels strongly depends on the local environment in interstellar clouds. They concluded that the atomic oxygen channel is the main route in translucent and diffuse clouds, while the molecular oxygen channel, together with the ozone route, is more efficient in dense cold molecular clouds. Water ice formation through surface reactions following $\mathrm{O}+\mathrm{H}, \mathrm{O}_{2}+\mathrm{H}$ and $\mathrm{O}_{3}+\mathrm{H}$ has been studied extensively and by different groups (Miyauchi et al. 2008; Ioppolo et al. 2008; Matar et al. 2008; Oba et al. 2009; Mokrane et al. 2009; Ioppolo et al. 2010a; Cuppen et al. 2010). Dulieu et al. (2010) used TPD in the submonolayer regime as their main analysis technique; the hydrogenation of solid $\mathrm{O}_{2}$ is the most extensively studied channel. Ioppolo et al. (2008, 2010a) investigated this reaction channel for a large range of astronomically relevant temperatures $(12-28 \mathrm{~K})$ (see Fig. 4). The main and surprising finding is that the initial formation rate of $\mathrm{H}_{2} \mathrm{O}_{2}$ and $\mathrm{H}_{2} \mathrm{O}$ is much less temperature dependent than the analogous reactions for $\mathrm{CO}$ hydrogenation. Furthermore, $\mathrm{O}_{2}$ hydrogenation results in a much larger yield than the few monolayers found for $\mathrm{CO}$ hydrogenation. This yield is strongly temperature dependent. Both effects are shown to be a direct consequence of the ability of $\mathrm{H}$ atoms to diffuse easily through an $\mathrm{O}_{2}$ ice (Ioppolo et al. 2010a). The experiments show that the penetration depth of $\mathrm{H}$ atoms into the ice depends strongly on the ice composition. In Ioppolo et al. (2010a) $\mathrm{O}_{2}$ hydrogenation is investigated extensively from a physical approach, i.e., studying different ice thicknesses, ice temperatures, ice structures and $\mathrm{H}_{2}$ concentrations in the atomic beam, whereas in a follow-up paper (Cuppen et al. 2010) the focus was on the reaction scheme with the intent to assess reaction routes and branching ratios. The latter work shows that the initially proposed reaction network by Tielens \& Hagen (1982) of only three mainly isolated channels is too simple and that several of the channels are actually linked through additional reactions. The third water formation channel (the hydrogenation of solid $\mathrm{O}_{3}$ ) was tested by Mokrane et al. (2009) and more recently by Romanzin et al. (2011). Since this channel is connected to the $\mathrm{O}_{2}$ channel after the first reaction step, special care was taken to deposit a pure $\mathrm{O}_{3}$ ice by keeping the substrate temperature between the $\mathrm{O}_{2}$ and $\mathrm{O}_{3}$ desorption temperature during deposition. If such a temperature is also kept during $\mathrm{H}$-atom addition, the $\mathrm{O}_{2}$ molecules formed upon $\mathrm{O}_{3}$ hydrogenation will desorb from the surface of the ice. In this way the reaction of $\mathrm{OH}$ to form water via $\mathrm{H}$ or $\mathrm{H}_{2}$ addition can be probed. The hydrogenation of $\mathrm{O}_{3}$ is found to behave more similar to $\mathrm{CO}$ hydrogenation in the sense that only the top few monolayers of $\mathrm{O}_{3}$ are hydrogenated. Moreover, the reaction $\mathrm{OH}+\mathrm{H}_{2}$ may be more efficient than the reaction $\mathrm{OH}+\mathrm{H}$ : reaction $\mathrm{OH}+\mathrm{H}_{2}$ likely proceeds through tunneling, while reaction $\mathrm{OH}+\mathrm{H}$ needs to dissipate $5.3 \mathrm{eV}$ of excess energy with just one final product, which could be difficult. These experimental results complete the reaction scheme on water formation initially proposed. The general conclusion that the three channels $\left(\mathrm{O} / \mathrm{O}_{2} / \mathrm{O}_{3}+\mathrm{H}\right)$ are strongly linked, is of importance for astrochemical models focusing on water formation under interstellar conditions (Wakelam et al. 2010). The recent identification of $\mathrm{H}_{2} \mathrm{O}_{2}$ in the interstellar medium (Bergman et al. 2011) is important within this context.

\subsection{Surface formation of $\mathrm{CO}_{2}$}

Ioppolo et al. (2011b) proved the surface formation of $\mathrm{CO}_{2}$ at low temperatures through the reaction $\mathrm{CO}+\mathrm{OH}$ and subsequent dissociation of the resulting $\mathrm{HO}-\mathrm{CO}$ complex 


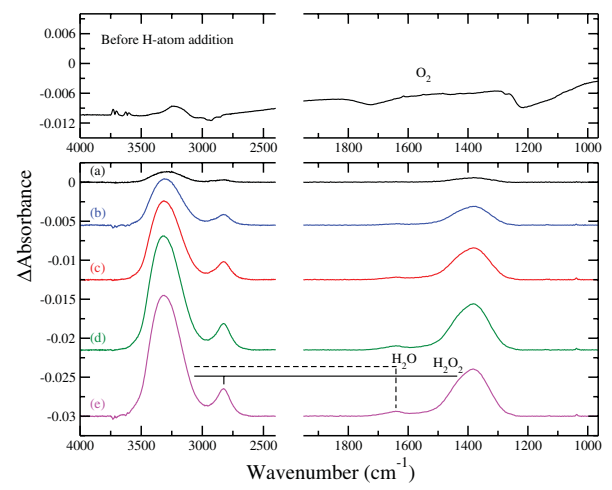

Figure 4. RAIR difference spectra of $\mathrm{O}_{2}+\mathrm{H}$ taken from (Ioppolo et al. 2010a). The top panel shows the $\mathrm{O}_{2}$ ice spectrum at $\mathrm{T}=15 \mathrm{~K}$. The bottom panels shows the RAIR difference spectra with respect to the initial deposited ice after $\mathrm{H}$-atom exposure at $\mathrm{T}=25 \mathrm{~K}$ for increasing fluences (a-e). The formation of $\mathrm{H}_{2} \mathrm{O}_{2}$ and $\mathrm{H}_{2} \mathrm{O}$ is clearly observed.

by hydrogenation of a $\mathrm{CO}: \mathrm{O}_{2}$ ice mixture. Such a binary ice is not fully representative for an interstellar ice, but the hydrogenation of $\mathrm{O}_{2}$ ice produces $\mathrm{OH}$ radicals, which allows the investigation of the interstellar relevant $\mathrm{CO}+\mathrm{OH}$ solid state reaction. As discussed above similar astrophysical ice studies have focused on the investigation of isolated surface reaction schemes, starting from the hydrogenation of pure ices, like solid $\mathrm{CO}$ and $\mathrm{O}_{2}$. For such ices no $\mathrm{CO}_{2}$ formation was observed upon $\mathrm{H}$-atom exposure. The hydrogenation of binary ice mixtures allows to investigate the influence of the presence of other species in the ice on the pure ice reaction schemes. Mixtures of $\mathrm{CO}: \mathrm{O}_{2}$ are deposited on a substrate under UHV conditions at low temperatures (15 and $20 \mathrm{~K}$ ) and are subsequently hydrogenated. The RAIRS shows that solid $\mathrm{CO}_{2}$ is formed in all studied $\mathrm{CO}: \mathrm{O}_{2}$ mixtures. Within the experimental uncertainties no dependency on ice temperature or composition was observed. The competition between $\mathrm{CO}$ and $\mathrm{O}_{2}$ hydrogenation in the mixed ice reveals that the penetration depth of $\mathrm{H}$ atoms into the ice depends strongly on the ice composition, and that the $\mathrm{CO}$ and $\mathrm{O}_{2}$ channels influence each others final product yields. The formation rate for all the final products is found to be less sensitive on the mixture composition than the final yield. Therefore, the formation rates found for $\mathrm{H}_{2} \mathrm{CO}, \mathrm{CH}_{3} \mathrm{OH}, \mathrm{H}_{2} \mathrm{O}_{2}$ and $\mathrm{H}_{2} \mathrm{O}$ in the isolated studies of the $\mathrm{CO}+\mathrm{H}$ and $\mathrm{O}_{2}+\mathrm{H}$ channels are valid for use in astrochemical models. In addition, surface $\mathrm{CO}_{2}$ formation without energetic input is found to be an important formation mechanism, which may explain the formation of $\mathrm{CO}_{2}$ together with $\mathrm{H}_{2} \mathrm{O}$ ice during the dense cold core phase prior to star formation. More details are available from Ioppolo et al. (2010b).

\section{VUV irradiation ices: photo desorption and photo processing}

The interstellar radiation field (ISRF) results in a background UV field of about $10^{8}$ photons $\mathrm{cm}^{-2} \mathrm{~s}^{-1}$. The UV field is however enhanced by orders of magnitude close to young stellar objects and even in environments protected from external UV (e.g., in cloud cores and disk mid-planes) due to cosmic ray interactions with hydrogen. Photoprocessing of ices may induce non-thermal desorption of surface molecules. It triggers the formation of complex species and thus offers a complimentary pathway towards molecular complexity in space. In fact, the photochemistry of icy grain mantles was suggested as a path towards chemical complexity more than three decades ago. Famous are the UV irradiation experiments on interstellar ice mixtures comprising of many different constituents and resulting in the formation of large organic compounds, among which are amino acids (Bernstein et al. 2002; Muñoz Caro et al. 2002; Ehrenfreund et al. 2001; 


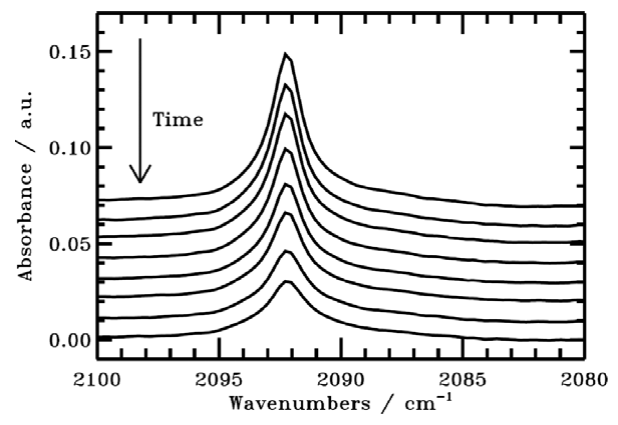

Figure 5. RAIR difference spectra of $\mathrm{C}^{18} \mathrm{O} \nu=1-0$ vibrational band at $2093 \mathrm{~cm}^{-1}$ acquired before irradiation of $8 \mathrm{ML}$ of $\mathrm{CO}$ ice and subsequently after every hour of 8-hr irradiation. The drop in integrated absorbance of the CO ice band is linear with UV irradiation time. The photodesorption rate for $\mathrm{C}^{16} \mathrm{O}$ is the same within the experimental uncertainty. Figure from Öberg et al. (2007).

Charnley et al. 1992). The latter work has set the scene, but more controlled experiments are needed to study molecule formation in situ and in real time.

In the laboratory interstellar UV radiation is typically simulated using the output of broadband hydrogen-microwave discharge lamps. These peak around $121.6 \mathrm{~nm}$ (Ly- $\alpha$ ) and cover 115 - $170 \mathrm{~nm}(7.5-10.5 \mathrm{eV})$ with a variable photon flux from roughly 1 to $10 \times 10^{13}$ photons $\mathrm{cm}^{-2} \mathrm{~s}^{-1}$ at the ice surface. Since this flux is much higher than that in space, special care has to be taken to extrapolate laboratory results to processes that occur in the ISM. In Leiden the ices are prepared in another UHV setup CRYOgenic Photoproduct Analysis Device (CRYOPAD) (Fuchs et al. 2006) in an identical way as described in the previous paragraph for the $\mathrm{H}$-atom addition reactions in SURFRESIDE. The ice is subsequently UV processed. Here we will discuss results using RAIRS to identify the formation and destruction of ice constituents and TPD as an additional tool to monitor desorption processes and ice evolution.

One of the major conclusions of a series of recent UV irradiation studies of CO, $\mathrm{N}_{2}$, $\mathrm{CO}_{2}, \mathrm{H}_{2} \mathrm{O}$ and $\mathrm{CH}_{3} \mathrm{OH}$ ice (Öberg et al. 2007, 2009a,b,c; Westley et al. 1995) is that photodesorption, i.e., the process in which a photon induces a non-thermal evaporation of one or several molecules is more efficient than has been assumed in the past. This is an important result which may also explain why for example gaseous CO can still be found in regions in space where it should have been fully frozen onto dust grains (Bergin et al. 2001).

$\mathrm{CO}$ ice photodesorbs non-dissociatively; the process is illustrated in Fig. 5. Here the decreasing CO RAIRS signal is shown as a function of irradiation time. With the known UV flux at the surface, the loss of signal with time can be converted into a photodesorption yield, as the signal can be translated into a number density. It is possible to derive a photodesorption yield which is in the order of $10^{-3}$ desorbed molecules per incident UV photon for CO. Very recently it was shown by Fayolle et al. (2011b) that this process is wavelength dependent. Experiments using the DESIRS beam line at SOLEIL showed that the $\mathrm{CO}$ photodesorption rate follows the $\mathrm{CO}$ electronic absorption pattern. In contrast to $\mathrm{CO}, \mathrm{CO}_{2}, \mathrm{H}_{2} \mathrm{O}$ and $\mathrm{CH}_{3} \mathrm{OH}$ dissociate upon $\mathrm{UV}$ irradiation and the fragments can recombine or photodesorb. If the fragments are in the surface layers, they either desorb directly from the excess energy provided by the photon or react with other radicals, which may still result in indirect photodesorption due to the release of energy. If the dissociated fragments remain in the ice mantle, the radicals can either become frozen in the ice matrix, diffuse through the ice to react with other radicals or recombine reforming the parent molecule. To constrain the photodesorption of the ice and thus determine the loss of precursor molecules into the gas phase rather than into photoproducts in the 


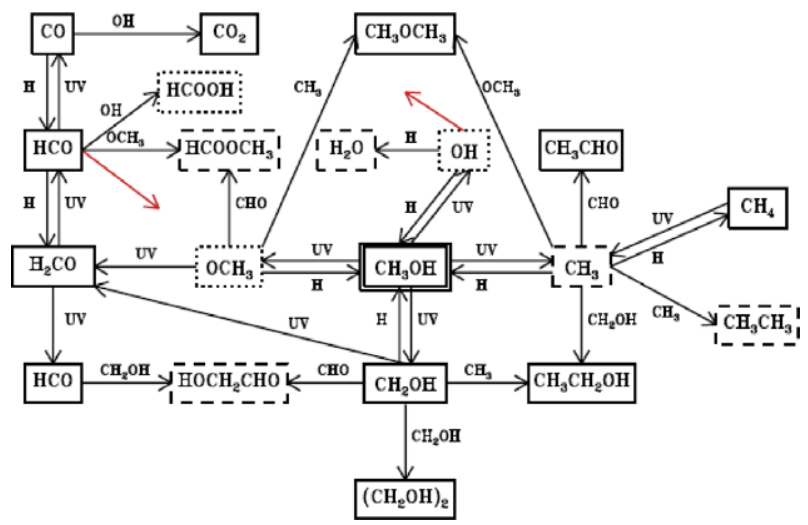

Figure 6. The proposed reaction scheme to form the observed products following UV-irradiation of pure $\mathrm{CH}_{3} \mathrm{OH}$ ice (solid boxes), of $\mathrm{CO}$ or $\mathrm{CH}_{4}: \mathrm{CH}_{3} \mathrm{OH}$ ice mixtures (dashed boxes) and products whose production could only be constrained with upper limits (dotted boxes).

ice, a special method has to be used. Photodesorption from a multilayer ice is a zeroth order process with respect to photon fluence, since it only depends on the amount of molecules in the surface layers. The photodesorption yield will not change with fluence as long as the original ice is sufficiently thick. Ice photolysis, however, is a first order process, since it depends on the total amount of ice. Through simultaneous modeling of the ice loss with an exponential decay and a linear function, the two processes can be separated, resulting in an effective photodesorption yield (Öberg et al. 2009a). For $\mathrm{CO}_{2}$ and $\mathrm{H}_{2} \mathrm{O}$ again values of the order of $10^{-3}$ photon ${ }^{-1}$ have been found, for $\mathrm{CH}_{3} \mathrm{OH}$ the photodesorption yield is about twice as large (Öberg et al. 2009c). The photodesorption of $\mathrm{N}_{2}$ ice has a yield that is at least an order of magnitude lower; $\mathrm{N}_{2}$ only co-desorbs with other molecules since it has no dipole-allowed transition within the energy range of the lamp. This observation is consistent with the recent wavelength dependent results found for CO (Fayolle et al. 2011b). It should be noted that not only the UV field, but also the ice thickness, ice temperature, and temperature dependent mean-free-path of the excited molecule play a role. Empirically derived photodesorption yields as function of these parameters are available from Wakelam et al. (2010).

In pure $\mathrm{CH}_{3} \mathrm{OH}$ ices $\mathrm{UV}$-induced photodissociation produces several different fragments - $\mathrm{CH}_{3}, \mathrm{CH}_{3} \mathrm{O}, \mathrm{CH}_{2} \mathrm{OH}, \mathrm{OH}$ - which can react to form complex organic molecules such as ethanol from $\mathrm{CH}_{3}$ and $\mathrm{CH}_{2} \mathrm{OH}$. This is illustrated in Fig. 6. Such reactions followed by ice desorption have been proposed to explain observations of gas-phase complex organic molecules in the warm inner regions of protostellar envelopes, so-called hot cores. In a series of dedicated RAIRS experiments, monitoring the UV photoproducts as a function of fluence, Öberg et al. 2009c produced all of the complex molecules commonly observed in hot cores (Bottinelli et al. 2007). Complementary TPD experiments have been used to further support identifications, since each product has a unique combination of mass signal and desorption temperature. The formation yields typically depend on ice temperature and ice composition as well as the UV flux and fluence. More details are available from Öberg et al. (2009d).

The role of the $\mathrm{H}_{2} \mathrm{O}$ concentration on the photochemistry of more volatile species was studied as well (Öberg et al. 2010). It was found, among others, that increasing the water concentration also increases the destruction efficiency of the volatile mixture constituent with a substantially higher trapping efficiency of the formed radicals in the ice. The formation of more complex species in this way is interesting since solid methanol has been observed towards a range of young stellar objects, including solar-type protostars. 
A UV driven methanol-ice chemistry may thus be a common pathway to chemical complexity in space. Complex molecule formation starting from methanol formation through CO hydrogenation and subsequent UV irradiation of methanol containing ices seems to provide a consistent reaction pathway for molecular complexity in space up to species with about 10 atoms. The presence of $\mathrm{H}_{2} \mathrm{O}$ may be essential as a catalyst.

At this stage, it should be noted that $\mathrm{H}$-atom bombardment and UV irradiation are not the only possibilities to trigger chemical reactions in an ice for astronomical conditions. Many efforts have been made, for example, to characterize the impact of ions on ice (Ioppolo et al. 2009; Palumbo 2006; Mennella et al. 2004; Baratta et al. 2002; Strazzulla et al. 2001). Thermal processing as well as the influence of electron and cosmic-ray impact on interstellar ice analogues have been studied (see e.g., Bennett et al. 2010). In recent years much progress has also been made in simulating the dynamical processes that take place in the ice (see e.g., Andersson \& van Dishoeck 2008; Arasa et al. 2010). This provides additional and valuable information to interpret laboratory observations in terms of interacting surface species.

\section{Complex molecules in ice}

From a laboratory perspective it is clear that complex molecules can be formed under astronomical conditions. The physical and chemical parameters, derived from work as described in the previous paragraphs, implemented into astrochemical models, indicate that solid state processes are relevant for the formation of larger species in space. A remaining question is which level of complexity can be reached in the ice. An alternative approach to answer this question is to skip the formation process in a top-down approach and to implement large complex molecules, such as polycyclic aromatic hydrocarbons (PAHs) directly into the ice. PAHs are found to be common throughout the interstellar medium, as evidenced by their (mid-IR) emission features that originate from vibrational relaxation after UV excitation. Towards dense clouds, however, these mid-IR features are strongly quenched. In these regions, as discussed before, most volatile molecules are frozen out on grains, forming layers of ice, and less volatile molecules such as PAHs are expected to condense on interstellar grains as well.

Previous spectroscopic ice studies focused on infrared experiments and have resulted in a large amount of spectroscopic data, also providing dynamical information (see e.g., Gudipati et al. 2003, 2006). In the specific case of PAHs, however, these have the disadvantage of spectral overlap; for different PAH members essentially the same vibrational modes are excited. This problem is partially overcome in the optical regime, since electronic energies will be different for different PAH species. Optical studies of photo-induced processes at play during the irradiation of $\mathrm{PAH}$ containing interstellar $\mathrm{H}_{2} \mathrm{O}$ ice analogues are available only for a few species and it was shown that these PAHs are readily ionized and stabilized within the ice, suggesting that trapped ions may play important, but overlooked roles in cosmic ice processes. Beyond this, there is little information on UV induced photochemistry and photophysics of $\mathrm{PAH}$ containing $\mathrm{H}_{2} \mathrm{O}$-rich ices.

A new measuring principle allows the spectroscopic and photo-dynamic study of PAH containing ice analogues upon UV irradiation, in situ and in real time (Bouwman et al. 2009). A high vacuum chamber is used in which ice is grown as described before, as well as a microwave hydrogen discharge source generating UV light for the photoprocessing, and a special Xe-arc light source emitting broad-band light that is transmitted through the ice onto a fast and fully computer controlled monochromator equipped with a sensitive CCD camera. This configuration results in a spectral coverage of 270 to $1200 \mathrm{~nm}$ and a time resolution down to $5 \mathrm{~ms}$ per spectrum. The solid precursor PAH species is brought into the gas phase through resistive heating and mixed with $\mathrm{H}_{2} \mathrm{O}$ vapor. A HeNe laser 
is used to determine the ice thickness of the mixture and in conjunction with optical spectroscopy on the PAH species this allows for an accurate concentration measurement. The innovative aspect of this setup is that it combines sensitive broadband spectroscopy with a resolution that is more than sufficient to monitor solid state features, i.e., it is possible to cover large spectral ranges without loss of spectral details, and a fast time response which allows monitoring ice chemistry in real time.

So far the UV induced photochemistry of the PAHs anthracene $\left(\mathrm{C}_{10} \mathrm{H}_{12}\right.$ or Ant), pyrene $\left(\mathrm{C}_{16} \mathrm{H}_{10}\right.$ or $\left.\mathrm{Py}\right)$, benzo[ghi]perylene $\left(\mathrm{C}_{22} \mathrm{H}_{12}\right.$ or $\left.\mathrm{BghiP}\right)$, and coronene $\left(\mathrm{C}_{24} \mathrm{H}_{12}\right.$ or Cor $)$ in $\mathrm{a} \sim 1$ :10,000 diluted $\mathrm{H}_{2} \mathrm{O}$ ice has been studied (Bouwman et al. 2009, 2010a,b, 2011a,b). The main conclusions of this work is that PAHs are likely to play a chemical role in the full solid state astrochemical solid state network.

\section{Outlook}

The richest inventory of complex organic molecules (COMs) has been found in a Galactic-center hot-core Sgr B2(N-LMH), including acetone, ethylene glycol, and glycolaldehyde. The temperature in these regions is $>100 \mathrm{~K}$ and molecules are in the gas phase. One of the major questions today is whether these COMs are first generation evaporated species produced by pure grain surface chemistry or second generation molecules formed by high temperature gas phase chemistry (after ice desorption). While several models suggest that the latter does not adequately explain the observed abundances (Wakelam et al. 2006), little quantitative information is available on the actual physical and chemical processes. More than 30 years ago experiments were performed in which ices containing typical astrophysical precursors were photo-processed with hard UV radiation (Agarwal et al. 1985; Hagen et al. 1979). The resulting organic refractory synthesized under high-vacuum conditions was extracted at room temperature and analysed by gas chromatography showing a rich variety of highly complex molecules. Whether these compounds were formed during UV irradiation of the ice sample or during the relatively intrusive analysis procedure could not be discriminated. New laboratory techniques with an emphasis on more delicate and sensitive sampling methods are therefore needed to tackle the subtle and complex chemistry taking place in the interstellar ice analogues. Limitations in IR spectroscopy, evidently suitable for simpler ices, become apparent when studying more complex ice mixtures. Overlapping IR absorption features and low detection sensitivity due to decreased state densities make the analysis difficult (one reason for studying PAHs with optical spectroscopy as described in the previous paragraph). The TPD mass spectroscopic analysis discussed before, requires the ice sample to be thermally evaporated, which may affect the chemical composition of the ice, and indefinitely implies the destruction of the ice sample. The inevitable prerequisite for mass spectroscopic analysis is the evaporation of the ice sample. An alternative for thermal desorption used in TPD is soft laser ablation - a technique typically used for non-volatile samples. In laser ablation, a short ns-UV laser pulse is focused onto the sample surface, where the photon energy is transferred to the sample leading to local desorption. MATRI2CES (Mass Analytical Tool for Reactions in Interstellar ICES) is a newly constructed setup in Leiden in which this technique has been implemented for the study of interstellar ice analogues. MATRI2CES combines laser ablation with time-of-flight mass spectroscopy, and is comparable to a standard MALDI-TOF setup. The freedom from having to heat-up and evaporate the ice as a whole means that the reactions in the ice analogues can be monitored in situ and online under astronomical conditions. Moreover, pulsed repetitive operation enables time-resolved monitoring of the chemical composition as well as depth-profiling of the ice. The construction of MATRI2CES was recently completed. 
To conclude: understanding the chemistry of complex organic molecules under interstellar conditions is particularly interesting due to their potential connection to pre-biotic chemistry. Is it possible to start with a realistic interstellar ice analogue and end up with an amino-acid? And if so, does this depend, for example, on temperature or the presence of specific constituents? How is this chemistry driven: atom bombardment, UV irradiation, a combination of both, or should other processes be considered? These are important questions that still have to be addressed, but in view of the progress obtained in the last years this is now work that can be dealt with in a systematic way in the nearby future.

\section{Acknowledgement}

The research topics highlighted in this proceeding have been obtained during several years of dedicated work by many $(\mathrm{PhD})$ students, postdocs and visitors and whose names are listed in the list with references. We are very thankful for their many contributions and discussions that have improved our understanding of solid state astrochemical processes. Financial support by NOVA (the Dutch Research School for Astronomy), NWO (the Netherlands Research Council), FOM (the Foundation for Fundamental Research of Matter), and the European Community's Seventh Framework Programme (FP7/20072013) under grant agreement no 238258 (LASSIE) is gratefully acknowledged.

\section{References}

Agarwal, V. K., Schutte, W., Greenberg, J. M., Ferris, J. P., Briggs, R., Connor, S., van de Bult, C. P. E. M., \& Baas, F. 1985, Origins of Life and Evolution of Biosphere, 16, 21

Andersson, S. \& van Dishoeck, E. F. 2008, A\& A, 491, 907

Arasa, C., Andersson, S., Cuppen, H. M., van Dishoeck, E. F., \& Kroes, G. 2010, JCP, 132, 184510

Baratta, G. A., Leto, G., \& Palumbo, M. E. 2002, A\&A, 384, 343

Bennett, C. J., Jamieson, C. S., Mebel, A. M., \& Kaiser, R. I. 2004, PCCP, 6, 735

Bennett, C. J., Jamieson, C. S., \& Kaiser, R. I. 2010, PCCP, 12, 4032

Bergin, E. A., Ciardi, D. R., Lada, C. J., Alves, J., \& Lada, E. A. 2001, ApJ, 557, 209

Bergman, P., Parise, B., Liseau, R., Larsson, B., Olofsson, H., Menten, K. M., \& Güsten, R. 2011, A\&A (Letters), 531,L8

Bernstein, M. P., Dworkin, J. P., Sandford, S. A., Cooper, G. W., \& Allamandola, L. J. 2002, Nature, 416, 401

Boogert, A. C. A., Pontoppidan, K. M., \& Knez, C. et al. 2008, ApJ, 678, 985

Bottinelli, S., Ceccarelli, C., Williams, J. P., \& Lefloch, B. 2008, Aध $A, 463,601$

Bottinelli, S., Boogert, A. C. A., \& Bouwman, J. et al. 2010, ApJ, 718, 1100

Bouwman, J., Paardekooper, D. M., Cuppen, H. M., Linnartz, H., \& Allamandola, L. J. 2009, ApJ, 700, 56

Bouwman, J., Cuppen, H. M., Bakker, A., Allamandola, L. J., \& Linnartz, H. 2010a, A $ళ$ A, 511, 33

Bouwman, J. 2010b, Spectroscopy and chemistry of interstellar ice analogues, Ph.D. Thesis, Leiden Observatory, Leiden University, P.O. Box 9513, 2300 RA Leiden, The Netherlands

Bouwman, J., Mattioda, A. L., Linnartz, H., \& Allamandola, L. J. 2011a, A\& A, 525, 93

Bouwman, J., Cuppen, H. M., Steglich, M., Allamandola, L. J., \& Linnartz, H. 2011b, A\&A, 529,46

van Broekhuizen, F. A., Pontoppidan, K. M., Fraser, H. J., \& van Dishoeck, E. F. 2005, A\&A, 441,249

Cazaux, S., Cobut, V., Marseille, M., Spaans, M., \& Caselli, P. 2010, A\&A, 522, A74

Charnley, S. B., Tielens, A. G. G. M., \& Millar, T. J. 1992, ApJ (Letters), 399, L71

Charnley, S. B., Tielens, A. G. G. M., \& Rodgers, S. D. 1997, ApJ (Letters), 482, L203

Charnley, S. B., Rodgers, S. D., \& Ehrenfreund, P. 2001, A\&A, 378, 1024

Charnley, S. B. \& Rodgers, S. D. 2005, in: D.C. Lis, G.A. Blake, E. Herbst (eds.), Astrochemistry: Recent Successes and Current Challenges, Proc. IAU Symposium No. 231 (Pacific Grove, California, USA), p. 237 
Cuppen, H. M., van Dishoeck, E. F., Herbst, E., \& Tielens, A. G. G. M. 2009, A\& A, 508, 275

Cuppen, H. M., Ioppolo, S., Romanzin, C., \& Linnartz, H. 2010, PCCP, 12, 12077

Cuppen, H. M., Pendeado, E. M., Isokoski, K. M., van der Marel, N., \& Linnartz, H., 2011, $M N R A S$, in press

Cuppen, H. M. \& Herbst, E. 2007, ApJ, 668, 294

Dulieu, F., Amiaud, L., Congiu, E., Fillion, J., Matar, E., Momeni, A., Pirronello, V., \& Lemaire, J. L. 2010, $A \mathscr{E} A, 512$, A30

Ehrenfreund, P., Bernstein, M. P., Dworkin, J. P., Sandford, S. A., \& Allamandola, L. J. 2001, ApJ (Letters), 550, L95

Fayolle, E. C., Öberg, K. I., Cuppen, H. M., Visser, R., \& Linnartz, H. 2011a, A\&A, 529, A73

Fayolle, E. C., Bertin, M., Romanzin, C., Michaut, X., Öberg, K. I., Linnartz, H., \& Fillion, J. H. 2011b, ApJ (Letters), in press

Fraser, H. J., Collings, M. P., Dever, J. W., \& McCoustra, M. R. S., 2004, MNRAS, 353, 59

Fuchs, G. W., Acharyya, K., \& Bisschop, S. E. et al. 2006, FaDi, 133, 331

Fuchs, G. W., Cuppen, H. M., Ioppolo, S., Bisschop, S. E., Andersson, S., van Dishoeck, E. F., \& Linnartz, H. 2009, A\&A A, 505, 629

Garrod, R., Park, I. H., Caselli, P., \& Herbst, E. 2006, FaDi, 133, 5

Geppert, W. D., Thomas, R. D., \& Ehlerding, A., et al. 2005, JPhCS, 4, 26

Gibb, E. L., Whittet, D. C. B. \& Schutte, W. A. et al. 2000, ApJ, 536, 347

Gibb, E. L., Whittet, D. C. B., Boogert, A. C. A., \& Tielens, A. G. G. M. 2004, ApJ (Suppl.), 151,35

Goumans, T. P. M., Uppal, M. A., \& Brown, W. A. 2008, MNRAS, 384, 1158

Gudipati, M. S. \& Allamandola, L. J. 2003, ApJ (Letters), 596, L195

Gudipati, M. S. \& Allamandola, L. J. 2006, ApJ, 638, 286

Hagen, W., Allamandola, L. J., \& Greenberg, J. M. 1979, Ap\&SS, 65, 215

Hiraoka, K., Sato, T., Sato, S., Sogoshi, N., Yokoyama, T., Takashima, H., \& Kitagawa, S. 2002, ApJ, 577, 265

Hudgins, D. M., Sandford, S. A., Allamandola, L. J., \& Tielens, A. G. G. M. 1993, ApJSS, 86, 713

Ioppolo, S., Cuppen, H. M., Romanzin, C., van Dishoeck, E. F., \& Linnartz, H. 2008, ApJ, 686, 1474

Ioppolo, S., Palumbo, M. E., Baratta, G. A., \& Mennella, V. 2009, A\&\&A, 493, 1017

Ioppolo, S., Cuppen, H. M., Romanzin, C., van Dishoeck, E. F., \& Linnartz, H. 2010a, PCCP, 12,12065

Ioppolo, S. 2011d, Surface formation routes of interstellar molecules - A laboratory study, Ph.D. Thesis, Leiden Observatory, Leiden University, P.O. Box 9513, 2300 RA Leiden, The Netherlands

Ioppolo, S., Cuppen, H. M., van Dishoeck, E. F., \& Linnartz, H. 2011a, MNRAS, 410, 1089

Ioppolo, S., van Boheemen, Y., Cuppen, H. M., van Dishoeck, E. F., \& Linnartz, H. 2011b, MNRAS, 413, 2281

Ioppolo, S., Cuppen, H. M., \& Linnartz, H. 2011c, Rend. Fis. Acc. Lincei, DOI 10.1007/s12210011-0135-3

Matar, E., Congiu, E., Dulieu, F., Momeni, A., \& Lemaire, J. L. 2008, A\&A, 492, 17

Mennella, V., Palumbo, M. E., \& Baratta, G. A. 2004, ApJ, 615, 1073

Miyauchi, N., Hidaka, H., Chigai, T., Nagaoka, A., Watanabe, N., \& Kouchi, A. 2008, CPL, 456, 27

Mokrane, H., Chaabouni, H., Accolla, M., Congiu, E., Dulieu, F., Chehrouri, M., \& Lemaire, J. L. 2009, ApJ (Letters), 705, 195

Muñoz Caro, G. M., Meierhenrich, U. J., \& Schutte, W. A. et al. 2002, Nature, 416, 403

Oba, Y., Miyauchi, N., Hidaka, H., Chigai, T., Watanabe, N., \& Kouchi, A. 2009, ApJ, 701, 464

Öberg, K. I., Fuchs, G. W., Awad, Z., Fraser, H. J., Schlemmer, S., van Dishoeck, E. F., \& Linnartz, H. 2007, ApJ (Letters), 662, L23

Öberg, K. I., Boogert, A. C. A., Pontoppidan, K. M., Blake, G. A., Evans, N. J., Lahuis, F., \& van Dishoeck, E. F. 2008, ApJ, 678, 1032

Öberg, K. I., van Dishoeck, E. F., \& Linnartz, H. 2009a, A\&SA, 496, 281

Öberg, K. I., Linnartz, H., Visser, R., \& van Dishoeck, E. F. 2009b, ApJ, 693, 1209 
Öberg, K. I., Garrod, R. T., van Dishoeck, E. F., \& Linnartz, H. 2009c, AËA, 504, 891

Öberg, K. I. 2009d, Complex processes in simple ices: laboratory and observational studies of gas-grain interactions during star formation, Ph.D. Thesis, Leiden Observatory, Leiden University, P.O. Box 9513, 2300 RA Leiden, The Netherlands

Öberg, K. I., Fayolle, E. C., Cuppen, H. M., van Dishoeck, E. F., \& Linnartz, H. 2009e, A\& A, 505,183

Öberg, K. I., van Dishoeck, E. F., Linnartz, H., \& Andersson, S. 2010, ApJ, 718, 832

Öberg, K. I., Boogert, A. C. A., Pontoppidan, K. M., van den Broek, S., van Dishoeck, E. F., Bottinelli, S. Blake, G. A., \& Evans, II, N. J. 2011, preprint (astro-ph/1107.5825),

Palumbo, M. E. 2006, A\&SA, 453, 903

Pontoppidan, K. M., Fraser, H. J., Dartois, E., Thi, W.-F., van Dishoeck, E. F., Boogert, A. C. A., d'Hendecourt, L., Tielens, A. G. G. M., \& Bisschop, S. E. 2003, A\&A A, 408, 981

Pontoppidan, K. M., Boogert, A. C. A., \& Fraser, H. J. et al. 2008, ApJ, 678, 1005

Romanzin, C., Ioppolo, S., Cuppen, H. M., van Dishoeck, E. F., \& Linnartz, H. 2011, JCP, 134, 084504

Strazzulla, G., Baratta, G. A., \& Palumbo, M. E. 2001, AcSpe, 57, 825

Tielens, A. G. G. M. \& Hagen, W. 1982, A\&\&A, 114, 245

Tielens, A. G. G. M., Tokunaga, A. T., Geballe, T. R., \& Baas, F. 1991, ApJ, 381, 181

Wakelam, V., Herbst, E., \& Selsis, F. 2006, A $\& A$, 451, 551

Wakelam, V., Smith, I. W. M., \& Herbst, E. et al. 2010, Space Sci. Revs, 156, 13

Watanabe, N., Nagaoka, A., Shiraki, T., \& Kouchi, A. 2004, ApJ, 616, 638

Watanabe, N. \& Kouchi, A. 2002, ApJ (Letters), 571, L173

Westley, M. S., Baragiola, R. A., Johnson, R. E., \& Baratta, G. A. 1995, Nature, 373, 405

Zasowski, G., Kemper, F., Watson, D. M., Furlan, E., Bohac, C. J., Hull, C., \& Green, J. D. 2009, ApJ, 694, 459

\section{Discussion}

SмIтH: I have two questions relating to the activation energies/barriers that you cited. 1) Are all degrees of freedom thermalized at the same temperature in these experiments?

2) Are the values of these barriers dependent on the nature of the ice and/or the way in which it is prepared?

LINNARTZ: 1) Yes. The best proof of this is that the $\mathrm{H}$ atoms being formed in a thermal cracking source, and subsequently thermalized to room temperature through collisions, instantaneously thermalize upon surface impact. Details of this process are available from Fuchs et al. (2009). 2) Yes. The values depend on ice morphology (e.g., amorphous vs. crystalline) but the values should not depend on the way the ice has been prepared. This is also confirmed by reproducible results for identical ice systems from different laboratories (i.e., different experimental setups).

ОвА: Some reactions energetically unfavourable are included in the reaction network to the formation of $\mathrm{H}_{2} \mathrm{O}$, e.g., $\mathrm{HO}_{2}+\mathrm{H}_{2} \rightarrow \mathrm{H}_{2} \mathrm{O}_{2}$ (endothermic with huge barrier). What is the energy source to promote the reactions (in laboratory)?

LINNARTZ: A detailed and extensive answer to this question can be found in "Water formation by surface $\mathrm{O}_{3}$ hydrogenation", C. Romanzin, S. Ioppolo, H. M. Cuppen, E. F. van Dishoeck, H. Linnartz, J. Chem. Phys. 134 (2011) 084504, in section IVc, where these reactions Eqs. 7 and 8) are discussed. See also Figure 5. 